\title{
QUANTIFYING THE VALUE OF RFID IN AIR CARGO HANDLING PROCESS: A SIMULATION APPROACH
}

\author{
Miao He \\ Jacqueline Morris \\ Tao Qin \\ IBM China Research Lab \\ Zhongguancun Software Park \#19, \\ 8 Dongbeiwang Road, Shangdi, 100193, Beijing, CHINA
}

\begin{abstract}
Air cargo customers demand deliveries in a timely manner because the cargo is usually high-value and/or perishable. Any delay at the airport may result in unmet customer demand, incur high inventory-in-transit cost and damage the quality of perishable commodities. Faced with these problems, an Asia-based airline has resorted to Radio Frequency Identification (RFID) to improve the efficiency of its air cargo handling process. Before implementation, we employ the simulation approach to quantify the benefits of RFID deployment. Our study shows that the RFID system can significantly reduce the total costs with the same timing, indicating that RFID technology is appropriate for the time-sensitive industrial and commercial practices.
\end{abstract}

\section{INTRODUCTION}

Due to increasing interconnection, interaction and integration among geographically disconnected regions, air cargo volume has been growing steadily. Table 1 shows air cargo weight handled by the world revenue air traffic from 1999 to 2008, reported by the International Civil Aviation Organization (ICAO). Naturally, the increasing volume demands higher efficiency in air cargo handling.

Table 1: Air cargo volume 1999 - 2008 (Source: ICAO Annual Report of Council 2008)

\begin{tabular}{c|llllllllll}
\hline Year & 1999 & 2000 & 2001 & 2002 & 2003 & 2004 & 2005 & 2006 & 2007 & 2008 \\
\hline Air cargo volume (million tons) & 28.1 & 30.4 & 28.8 & 31.4 & 33.5 & 36.7 & 37.6 & 39.8 & 41.8 & 40.5 \\
\hline
\end{tabular}

In weight, airborne freights can hardly compare to the oceanborne freights, which usually measure in billions of tons. However, the proportion of air cargos in value cannot be neglected. According to a report published by the Research and Innovative Technology Administration (RITA) in 2002, air trade accounts for only $0.4 \%$ of all U.S. trade in weight but $25.1 \%$ in value.

These statistics indicate that air cargo timing is extremely important to the transport of time-sensitive and high-value goods. A delay or error at the airport may result in unmet customer demand, and incur storage or deterioration costs. Therefore, both the increasing volumes and the unique characteristics (high-value and time-sensitive) of air cargo call for highly efficient handling systems with low error rate.

This paper studies the air cargo handling process at an Asia-based airline. The corporation operates services at 53 domestic and 25 international destinations. From 2005 to 2009, the air freight revenue steadily accounts for around $5 \%$ of domestic operations, and approximately $20 \%$ of international operations.

The airline is considering to apply Radio Frequency Identification (RFID) technology to improve its operational efficiency. RFID technology refers to the use of radio waves to identify or track a product, animal or purpose with a microchip (typically referred to as an RFID tag). Some tags can be read from several meters away and beyond the line of sight of the reader. RFID has the capability of accessing multiple tags simultaneously, even when the tags are attached to high-speed objects. RFID also outperforms humans in 
identification accuracy. Consequently, it eliminates quite a proportion of re-worked cargos and removes delays resulting from inaccuracies in the handling process. In this paper, we apply simulation approach to validate the benefits of adopting RFID.

The rest of the paper is organized in the following manner: Section 2 reviews the literature related to air cargo handling and RFID; Section 3 maps the real-world system and formulates a discrete-event simulation model. Section 4 describes the methodology used to study the problem and reports the numeric results from simulation. We discuss future research topics in Section $\mathbf{5}$.

\section{LITERATURE REVIEW}

Air cargo shipping and handling is an important part of logistics service. Several observations have been made regarding to its efficiency. According to Chen and Chou (2006), 91\% of the time taken to ship air cargo was spent on the ground in 1961, and the air cargo shipment took on average six days to arrive for customers in 1988. Lobo and Zairi (1999) found that an air cargo shipment passed through about 40 hands and was associated with 12 documents from the shipper to the customer.

Researchers have been studying how to increase the efficiency of air cargo handling. Delorme (1992) detailed a simulation model, including input and output, of the air cargo handling system at American Airlines Cargo (AA Cargo). The simulation model was first used to evaluate the effectiveness of existing operating procedures and resource allocations, and second, to quantify the impact of proposed modifications in layout, procedures and resource allocations. In more recent work, Nsakanda, Turcotte, and Diaby (2004) developed a simulation model to quantify the performance of air cargo handling operations under different shipment storage policies. They also answered the what-if questions when the processing times changed, or when some equipment broke down. Khan (2000) applied Harbour's seven-step process improvement method to the air cargo handling process, and reduced total cycle time from 4875 mins to 1265 mins via the business process reengineering.

In addition to research targeting the overall air cargo handling process, there are also studies specifically focusing on the automatic storage and retrieval system (AS/RS) that temporarily stores air cargo during the handling process. Lee et al. (2005) proposed a class-based space reservation storage policy to minimize the expected $\mathrm{S} / \mathrm{R}$ machine travel time given uncertain cargo arrival and storage time. In a later paper (Lee et al. 2006), they further incorporated the airline assignment into their model and used simulation to evaluate the airline assignment policy and the AS/RS storage policy. Other air cargo related topics involve revenue management (Amaruchkul, Cooper, and Gupta 2007), air cargo terminal design (Chen and Chou 2006) and air cargo goodsflow control (Verwijmeren and Tilanus 1993).

Advanced technologies, such as RFID and the Internet of Things (IoT), can bring significant changes to the handling process. RFID technology is applicable to a wide range of scenarios (Ferrer, Dew, and Apte 2010). Out of its many uses, the ability to provide real-time data is well-suited for logistics and supply chain management with RFID. Chow et al. (2006) designed an RFID-based resource management system to help users select the most suitable resource packages for handling warehouse operations. Angeles (2005) used seven case examples to demonstrate the benefits of implementing RFID in the supply chain. Further, with smart objects connected to the Internet, IoT can upgrade the capabilities of RFID with faster identification, higher accuracy and more visibility. IoT also installs services and intelligence on top of smart objects. The embedded intelligence can support business processing decomposition and distributed execution (Haller, Karnouskos, and Schroth 2009). Capturing more and more attention recently, IoT can radically change the way we live (Gershenfeld, Krikorian, and Cohen 2004) and the way we operate our business (Bose and Pal 2005).

In our application, the airline adopts RFID, and we quantify the benefits from the implementation. Previous research is available with regard to quantifying the benefits of RFID. Karaer and Lee (2007) compared among three types of manufacturers: the naive manufacturer who does not realize the impact of reverse channel or information, the enlightened manufacturer aware of his reserve channel without visibility and lastly, the manufacturer with full visibility to his supply chain. They then computed the savings in the reverse supply chain resulting from the full visibility endowed by RFID. Hou and Huang (2006) used empirical study to reveal the characteristics of the printing industry and classified the supply chain into six fundamental business models. The costs and benefits (time savings) of RFID application were calculated for each model based on their business scenarios. Besides these quantitative studies, other research (Tzeng, Chen, and Pai 2008, Soon and Gutiérrez 2008) has explored the qualitative impact of implementing RFID-enabled systems.

We use the simulation approach to accomplish the quantification, which is widely adopted in many contexts, including modeling natural systems or human systems to gain insight into their functioning (Aldrich 2005). Law (2007) values simulation as one of the most widely used operations-research and management-science 


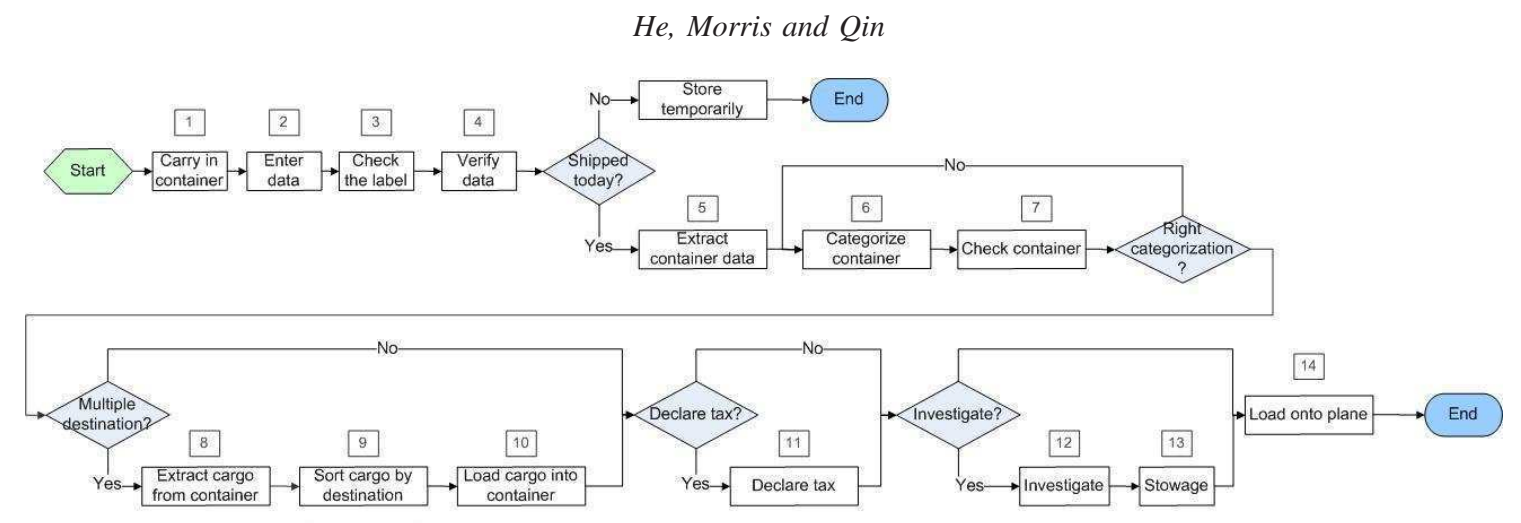

Figure 1: The AS-IS system map

techniques. Much simulation-enabled research is readily available for the logistics and supply chain management. (Disney, Naim, and Towill 1997, Legago and Mazza 2001).

\section{MODELING: AS-IS SYSTEM AND TO-BE SYSTEM}

We model the complete RFID deployment, what we will call the TO-BE system, in comparison to the now in place AS-IS system. Figure 1 shows main procedures that an arriving container goes through in the current system. After carrying in the container, the operators need to enter container information, including M-AWB (Master Air Way Bill) number, H-AWB (House Air Way Bill) number, destination information etc. The operators will then check and verify that the information on the label is consistent with that in the logistics system. Then goods scheduled to be shipped on that day will enter the subsequent procedures, while the others are put into temporary storage.

For goods scheduled to ship on the arriving day, the operators will first extract their information from the logistics system, and categorize them according to their attributes, e.g. number of destinations, the necessity of any tax declaration, and the necessity of investigation. The categorization will determine the loading arrangements of the goods. For example, if a container contains goods for both Destination A and B, and A must be visited before B, it will enter the "Yes" branch in the decision node of "Mutiple Destination?" in Figure 1, and the cargo will be sorted. Goods to Destination A will be put above those to Destination B for unloading convenience. Conversely, no sorting is required if all the goods in a container are going to the same destination, i.e., and enter the "No" branch. Incorrectly categorized containers will reenter the sorting procedure.

Note that the arriving day may not be the scheduled shipping day. In such case, steps 1-4 are performed on the arriving day and steps 5-14 on the shipping date.

In the TO-BE system, after data entry (the second step), the operators will create and attach RFID tags to containers, which replace the two steps of label checking and data verification. With the convenience of RFID, we significantly increase the speed of data extraction and cargo categorization, i.e. the fifth and sixth steps. Now, instead of manually reading the labels and categorizing the goods, a computer can sort the RFID identification tags by destination, and dictate the arrangement of the shipments in a faster and more accurate manner. Even better, RFID readers can retrieve data from multiple tags at the same time.

\subsection{Value Propositions of RFID}

We notice three major benefits created by RFID in our case: increasing cargo visibility, streamlining the handling process and standardizing the procedures. The increased visibility enables real-time access to cargo data and attains higher monitor accuracy. Human errors and labor hours are reduced after streamlining the process with RFID. And by standardizing the RFID-related procedures, the better operation quality will improve the service quality. Table 2 summarizes the benefits of RFID implementation in the air cargo handling process. 


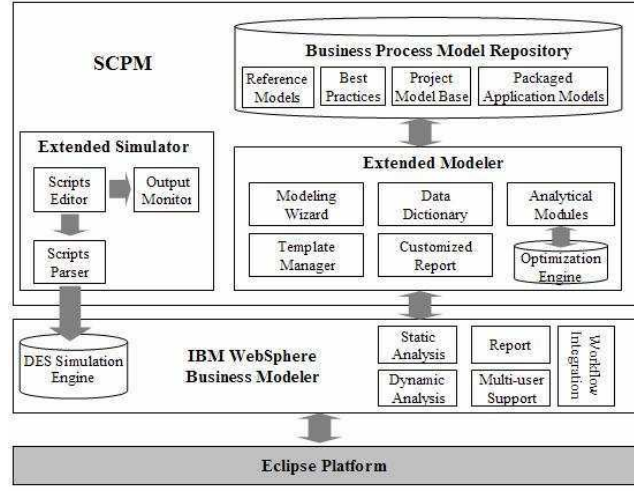

(a) SCPM architecture

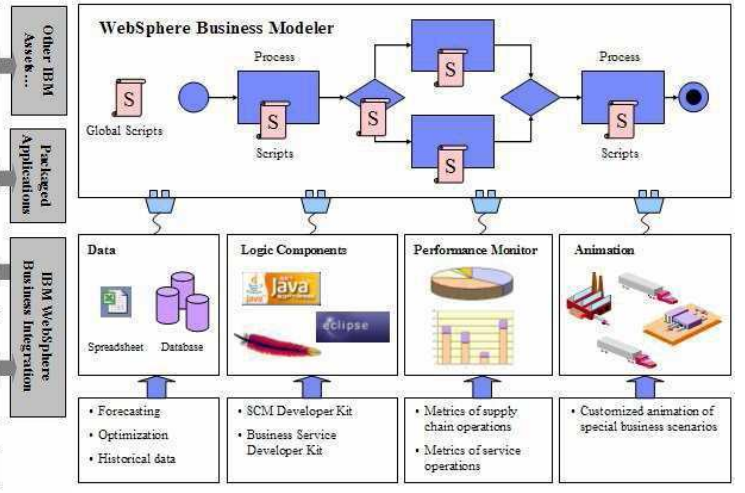

(b) Script-based simulation mechanism

Figure 2: IBM Supply Chain Process Modeler

Table 2: Values of RFID in air cargo handling process

\begin{tabular}{l|l|c|c|c}
\hline \multirow{2}{*}{ Value } & \multirow{2}{*}{ Merit } & \multicolumn{3}{|c}{ Benefits } \\
\cline { 2 - 4 } & & Quality & Time & Cost \\
\hline \multirow{3}{*}{ Visibility } & Monitor cargo status & $\mathrm{O}$ & $\mathrm{O}$ & \\
\cline { 2 - 5 } & Improve cargo control accuracy & $\mathrm{O}$ & $\mathrm{O}$ & $\mathrm{O}$ \\
\cline { 2 - 5 } & Automatically computerize cargo data & $\mathrm{O}$ & $\mathrm{O}$ & \\
\hline \multirow{3}{*}{ Streamlining } & Reduce human errors & $\mathrm{O}$ & $\mathrm{O}$ & $\mathrm{O}$ \\
\cline { 2 - 5 } & Reduce labor hours & & $\mathrm{O}$ & \\
\cline { 2 - 5 } & Increase throughput & & & $\mathrm{O}$ \\
\hline \multirow{2}{*}{ Standardization } & Improve service quality & & $\mathrm{O}$ & $\mathrm{O}$ \\
\cline { 2 - 5 } & Simplify the process & & & \\
\hline
\end{tabular}

\subsection{The Tool: Supply Chain Process Modeler}

We use IBM Supply Chain Process Modeler (SCPM) to develop and implement our simulation models. SCPM seamlessly "plugs in" another IBM standard software Websphere Business Modeler, which "enables users to model, design, simulate, analyze and generate reports for business process, integrate new and revised process, and define organizations, resources and business items" (Wahli et al. (2006)).

SCPM has struck a balance between usability and flexibility. From the usability perspective, SCPM provides a generic reference model repository, which stores standardized cross-industry models such as the SCOR (Supply Chain Operations Reference) model (SCC 2009) and APQC PCF (American Productivity and Quality Center Process Classification Framework) (APQC 2009). The reference models offer insight into business processes and best practices. This helps modelers think through the processes and model them in an structural way. Figure 2(a) outlines the architecture of SCPM.

From the flexibility perspective, SCPM extends WBM with script-based business process simulation. The simulation script is very similar to the standard Java language. Users can easily code within processes and decision nodes. The simulation engine supports script execution before and after the process execution. It also accepts customized delay, cost, revenue and duration at each process. The embedded script allows modeling complicated systems, complex decision rules and even calling for external components such as optimization engines. This results in a highly extensible tool. Figure 2(b) depicts the script-based mechanism.

Figure 3 is a screenshot of our modeler. The upper left panel could navigate modelers to different projects or different processes within the same project. The upper right panel is the main modeling area where we can easily drag and drop predefined elements to compose a whole model. The lower left panel is an outline of the complete model on the modeling area, with which we can fast position to the modeling subarea we want to edit. When in need of complicated logic, complex decision rules and/or links to external component, we can write scripts as shown in the lower right panel. 


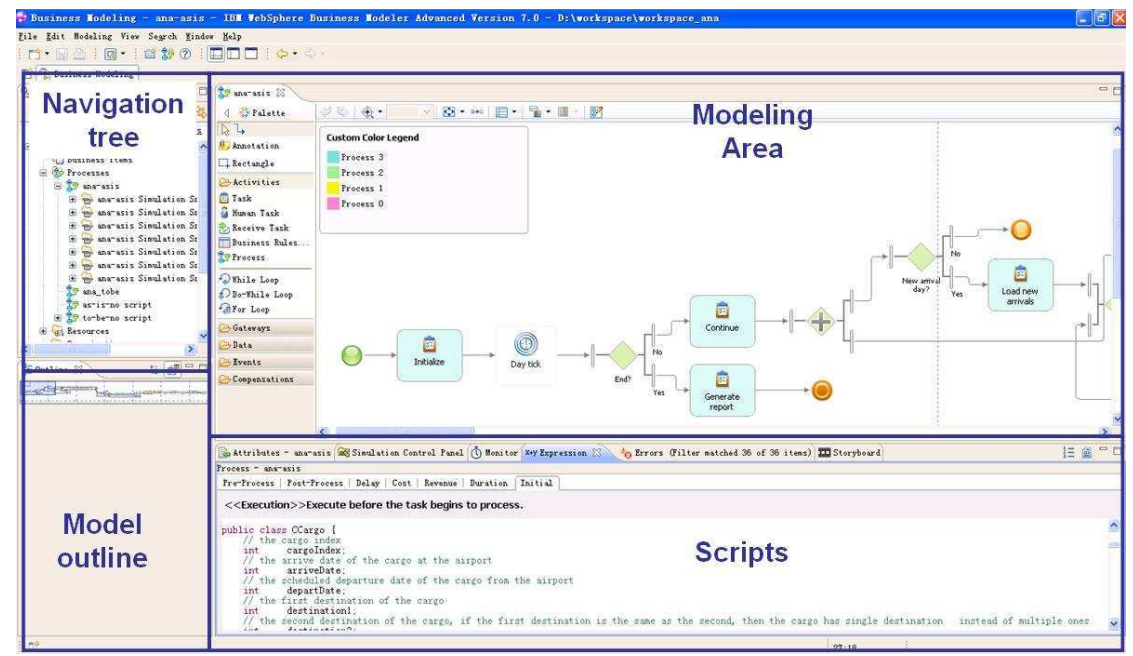

Figure 3: The AS-IS system map
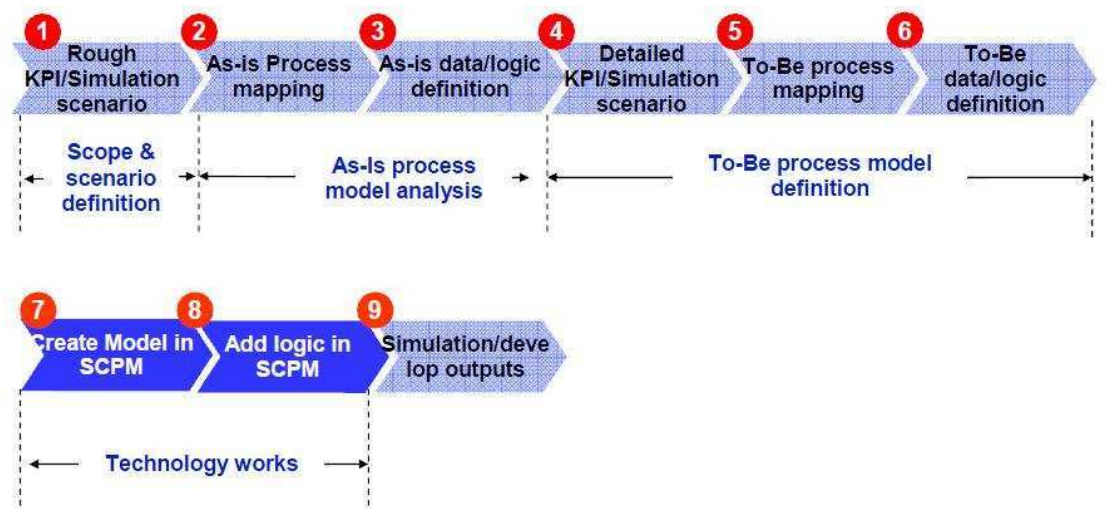

Figure 4: Methodology of simulation-enabled consultancy project

\section{METHODOLOGY AND NUMERIC RESULTS}

We will first describe our step-by-step methodology to accomplish a simulation-based consultancy project with SCPM, and then summarize the results of numeric experiments.

\subsection{Methodology}

We have developed a nine-step methodology, shown in Figure 4, to facilitate the simulation-enabled consultancy project with SCPM. Effective collaboration between business consultants and the technical team is essential for successful SCPM model creation. The consulting team focuses on project scoping, simulation scenarios and key performance indicators (KPIs) definitions, conceptualizing AS-IS and TO-BE models and logic, collecting data, and analyzing simulation results. The technical team focuses on developing simulation models and scripts, running simulations, and providing feedback to the consulting team. Ideally, the technical team should be involved at the start of the project to ensure better understanding and control.

\subsection{Numeric Results}

We simulate 30 days and generate 1000 cargos on each day with random attributes, including the number of destinations, the scheduled shipping date, the tax declaration requirement and the investigation requirement. 


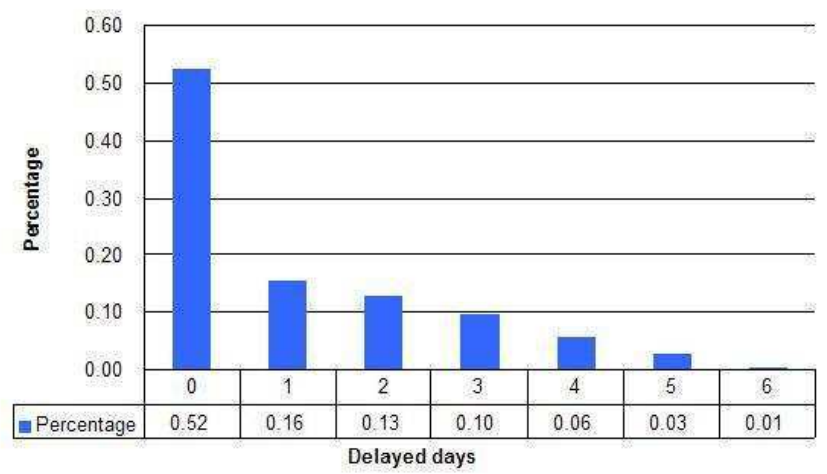

Figure 5: The percentage of delayed days with 4 operators at each step

We argue that the first several days in the simulation have nonstationary input of earlier arriving cargos and should be excluded from the numeric results. Moreover, there are seldom cargos arriving at the airport that have to wait more than ten days for shipping. We therefore define the first ten days as the warming period in the simulation.

\subsubsection{Processing Time Changes and Delayed Days}

In the AS-IS system, five operators are installed at each processing step. Notice that step 3 and step 4 will be replaced by "create RFID tags" and "attach RFID tags" in the TO-BE system, eliminating the labor requirement in steps 5 and 6 as a result of RFID implementation.

The total processing time is composed of two parts: the processing time from step 1 to step 4, and the time from step 5 to 14 . Note that the queueing time is also included into the total processing time. In Table 3 we summarize the total processing time for the AS-IS system and the TO-BE system with 4, 5, and 6 operators at each step, respectively. The percentages of delayed cargos are also summarized in Table 4.

We observe that configuring 5 operators at each step in the AS-IS system just meets the demand. While the current system is sufficient with 5 operators, removing one operator at each step will cost on average more than six times the processing time and delay up to $48 \%$ of the cargo. Under the 4-operator assumption, Figure 5 displays the percentage of cargo suffering from a delay, which may vary from zero to six days.

These numeric experiments alert the airline that although the AS-IS system luckily satisfies the current demand, it may suffer from congestions resulting from operator absence or increasing demand. In contrast, the TO-BE system with 4 operators per step achieves approximately equivalent performance as the AS-IS system with 5 operators per step. Retaining the 5-operator configuration in the TO-BE system offers buffers and flexibility to accommodate emergency cases. We also emphasize that under the same operator configuration, the TO-BE system achieves smaller variance than the AS-IS system in processing time(see the standard deviation row in Table 3).

The better performance of the TO-BE system is two-fold: on one hand, because an RFID reader can access more than 50 tags simultaneously, it does the work of 50 operators. This is ten times faster than a 5-operator system. On the other hand, the faster data access speed of an RFID reader shortens the data extraction time of step 5 from one minute to almost zero seconds, and reduces the cargo categorization time of step 6 from 30 seconds to almost zero seconds for each tag.

Table 3: Total processing time comparison: As-is system versus TO-BE system

\begin{tabular}{l|c|ccc}
\hline \multirow{2}{*}{ System } & & \multicolumn{3}{|c}{ Total processing time (hour) } \\
\cline { 3 - 5 } & & 4 operators & 5 operators & 6 operators \\
\hline \multirow{2}{*}{ As-is } & Mean & 107.80 & 17.95 & 14.54 \\
& Stdev & 34.75 & 4.34 & 4.14 \\
\hline \multirow{2}{*}{ To-be } & Mean & 17.11 & 13.67 & 11.49 \\
& Stdev & 4.35 & 3.49 & 2.93 \\
\hline
\end{tabular}


He, Morris and Qin

Table 4: Percentage of delayed cargos: As-is system versus TO-BE system

\begin{tabular}{l|ccc}
\hline \multirow{2}{*}{ System } & \multicolumn{3}{|c}{ Percentage of delayed cargos } \\
\cline { 2 - 4 } & 4 operators & 5 operators & 6 operators \\
\hline As-is & $48 \%$ & $0 \%$ & $0 \%$ \\
\hline To-be & $0 \%$ & $0 \%$ & $0 \%$ \\
\hline
\end{tabular}

\subsubsection{Return on Investment (ROI)}

Table 3 shows that in terms of both mean and standard deviation of cargo processing time, the TO-BE system with 4 operators at each step are comparable to the AS-IS system with 5 operators at each step. With comparable performance, we next quantify the expected savings of the 4-operator TO-BE system over the 5-operator AS-IS system.

Table 5: Parameter setting for the ROI calculation

\begin{tabular}{|c|c|c|c|c|c|c|}
\hline \multirow{2}{*}{ System } & \multicolumn{2}{|c|}{ Operators } & \multicolumn{2}{|c|}{ RFID readers } & \multicolumn{2}{|c|}{ RFID tags } \\
\hline & Quantity & $\begin{array}{c}\text { Price(/day } * \\
\text { person) }\end{array}$ & Quantity & Price(/item) & Quantity & Price(/item) \\
\hline As-is & $70 * 3$ & $171 \$^{(1)}$ & 0 & $3500 \$$ (2) & 0 & $0.5 \$^{(3)}$ \\
\hline To-be & $48 * 3$ & & 2 & & 1000 & 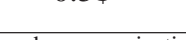 \\
\hline
\end{tabular}

We will average the RFID cost over its life time, and obtain the annual costs before and after installing the RFID readers. The empirical life span for an RFID reader is four years. The annual cost before the RFID implementation is only for labor, about 13.11 million USD ( $\$ 171$ /operator $*$ day $* 70$ operators/shift $* 3$ shifts/day $* 365$ days/year). After the improvement, the total costs includes labor costs, RFID reader costs, and RFID tag costs. With the TO-BE system, labor costs decrease to about 8.99 million USD. The RFID reader costs about $\$ 1750$ per year, and RFID tags costs \$ 0.5/tag * 1000 tags/day * 365 days/year (0.18 million USD). Ultimately, the RFID system will save the airline 3.94 million USD (or 30\%) per year in the air cargo handling process. Note that we have not sacrificed time efficiency of air cargo handling process with the significant cost reduction.

\section{FUTURE RESEARCH}

In this paper, we present a simulation analysis to quantify the impact of adopting RFID in the air cargo handling process for an Asia-based airline. The numeric results not only show the robustness and flexibility of an RFID system to tackle increasing demand and unforseen events such as operator absence, but also provide a way to estimate the cost reduction (as much as 30\%), which helps make evidence-based and data-driven decisions.

Our model could always be improved to be more realistic, systemic and optimized. One potential extension is to incorporate the airline scheduling. We currently assume the cargo bears no delay if the handling process ends on the scheduled departure date, but it is quite possible that there are no scheduled flights after the end of cargo handling. In such a circumstance, our model needs to be updated to interact with airline scheduling module. Moreover, the number of operators at each step does not need to remain the same but can be further optimized according to the processing times required. Finally, we also point out that when we calculate the ROI, we have not covered all possible costs. For example, we have not included the training costs of using RFID readers, the possible repair costs for broke RFID equipments, and the costs of management team, etc. Incorporating these costs into the model can analyze the value of RFID implementation more accurately.

If the Internet of Things (IoT) becomes a reality, the system can be more efficient because it will enable automatic tax declaration (step 11) and may even eliminate the data entry procedure (step 2). With the assistance of new technologies and techniques from the operations research society, we can expect an air cargo handling system with higher efficiency and more satisfied customers.

\section{REFERENCES}

Aldrich, C. 2005. Learning by doing: A comprehensive guide to simulations, computer games and pedagogy in e-learning and other educational experiences. John Wiley and Sons. 
Amaruchkul, K., W. L. Cooper, and D. Gupta. 2007. Single-leg air-cargo revenue management. Transportation Science 41 (4): 457-469.

Angeles, R. 2005. RFID technologies: Supply-chain applications and implementation issues. Information Systems Management 22 (1): 51-65.

Bose, I., and R. Pal. 2005. Auto-id: Managing anything, anywhere, anytime in the supply chain. Communications of the ACM 48 (8): 100-106.

Chen, C.-H., and S.-Y. Chou. 2006. A bsc framework for air cargo terminal design: Procedure and case study. Journal of Industrial Technology 22 (1): 1-10.

Chow, H. H. K., K. L. Choy, W. B. Lee, and K. C. Lau. 2006. Design of a RFID case-based resource management system for warehouse operations. Expert Systems with Applications 30 (4): 561-576.

Delorme, P. 1992. Simulation of a combination carrier air cargo hub. Proceedings of the 1992 Winter Simulation Conference: $1325-1331$.

Disney, S. M., M. M. Naim, and D. R. Towill. 1997. Dynamic simulation modelling for lean logistics. International Journal of Physical Distribution \& Logistics 27 (3/4): 174-196.

Ferrer, G., N. Dew, and U. Apte. 2010. When is RFID right for your service? International Journal of Production Economics 124 (2): 414-425.

Gershenfeld, N., R. Krikorian, and D. Cohen. 2004. The internet of things. Scientific American 291 (4): 76-81.

Haller, S., S. Karnouskos, and C. Schroth. 2009. The internet of things in an enterprise context. Proceedings: The First Future Internet Symposium:14-28.

Hou, J.-L., and C.-H. Huang. 2006. Quantitative performance evaluation of RFID applications in the supply chain of the printing industry. Industrial Management \& Data Systems 106 (1): 96-120.

Karaer, O., and H. L. Lee. 2007. Managing the reverse channel with RFID-enabled negative demand information. Production and Operations Management 16 (6): 625-645.

Khan, M. R. R. 2000. Business process reengineering of an air cargo handling process. International Journal of Production Economics 63 (1): 99-108.

Law, A. M. 2007. Simulation modeling \& analysis. McGraw-Hill.

Lee, C., H. C. Huang, P. Goldsman, B. Liu, and Z. Xu. 2005. Researvation storage policy for as/rs at air cargo terminals. Proceedings of the 2005 Winter Simulation Conference:1627-1632.

Lee, C., H. C. Huang, P. Goldsman, B. Liu, and Z. Xu. 2006. Development of timed colour petri net simulation models for air cargo terminal operations. Computers \& Indusstrial Engineering 51 (1): 102-110.

Legago, P., and R. M. Mazza. 2001. Berth planning and resource optimisation at a container terminal via discrete event simulation. International Journal of Physical Distribution \& Logistics 133 (3): 537-547.

Lobo, I., and M. Zairi. 1999. Competitive benchmarking in the air cargo industry: Part i. Benchmarking: An International Journal 6 (2): 164-191.

Nsakanda, A. L., M. Turcotte, and M. Diaby. 2004. Air cargo operations evaluation and analysis through simulation. Proceedings of the 2004 Winter Simulation Conference:1790-1798.

Soon, C.-B., and J. A. Gutiérrez. 2008. Effects of the RFID mandate on supply chain management. Journal of Theoretical and Applied Electronic Commerce Research 3 (1): 81-91.

Tzeng, S.-F., W.-H. Chen, and F.-Y. Pai. 2008. Evaluating the business value of RFID: Evidence from five case studies. International Journal of Production Economics 112 (2): 601-613.

Verwijmeren, M. A. A. P., and C. B. Tilanus. 1993. Network planning for scheduling operations in air cargo handling: A tool in medium term goodsflow control. European Journal of Operational Research 70 (2): 159-166.

Wahli, U., L. Leybovich, E. Prevost, R. Scher, A. Venancio, S. Wiederkom, and M. N.. 2006. Business process management: Modeling through monitoring using websphere v6 products. Available at <http://www.redbooks.ibm.com/ >.

\section{AUTHOR BIOGRAPHIES}

MIAO HE is a Researcher at IBM China Research Laboratory. She joined IBM Research in 2009 after receiving her M.S degree in Industrial Engineering from Tsinghua University in Beijing, P. R. China. Her research interests include supply chain management, business process management, clinical decision making and stochastic dynamic programming. Her email address is <hmhem@cn.i.bm.com>.

JACQUELINE MORRIS is majoring in mathematics and economics at Barnard College, Columbia University. She is currently a research intern at IBM China Research Laboratory. Her email address is $<j m 2955 @$ Columbia. edu>.

TAO QIN is a Researcher at IBM China Research Laboratory. He joined IBM Research in 2010 after receiving his M.S. degree in Industrial Engineering from Pusan National University in Pusan, South Korea. Before joining IBM, he was a researcher at the Institute of Automation, Chinese Academy of Sciences, P. R. China. His research interests include port logistics system modeling, optimization and simulation. His e-mail address is <qtqint@cn.ibm.com>. 